\title{
A competitive enzyme immunoassay for the quantitative detection of cocaine from banknotes and latent fingermarks
}

\author{
Susan van der Heide ${ }^{\mathrm{a}}$, Paula Garcia Calavia ${ }^{\mathrm{a}}$, Sheila Hardwick ${ }^{\mathrm{b}}$, Simon Hudson ${ }^{\mathrm{c}}$, Kim \\ Wolff $^{d}$ and David A. Russella* \\ a School of Chemistry, University of East Anglia, Norwich Research Park, Norwich, Norfolk; \\ NR4 7TJ, UK. \\ ${ }^{\mathrm{b}}$ Home Office Centre for Applied Science and Technology (CAST), Sandridge, St. Albans, \\ Herts, AL4 9HQ, UK. \\ ' HFL Sports Science, LGC Health Sciences, Newmarket Rd, Fordham, Cambridgeshire, \\ CB7 5WW, UK. \\ d Institute of Pharmaceutical Science, Department of Pharmacy, King's College London, 150 \\ Stamford St, London, SE1 9NH, UK.
}

\begin{abstract}
A sensitive and versatile competitive enzyme immunoassay (cEIA) has been developed for the quantitative detection of cocaine in complex forensic samples. Polyclonal anti-cocaine antibody was purified from serum and deposited onto microtiter plates. The concentration of the cocaine antibody adsorbed onto the plates, and the dilution of the cocaine-HRP hapten were both studied to achieve an optimised immunoassay. The method was successfully used to quantify cocaine in extracts taken from both paper currency and latent fingermarks. The limit of detection (LOD) of $0.162 \mathrm{ng} \mathrm{mL}^{-1}$ achieved with the assay compares favourably to that of conventional chromatography-mass spectroscopy techniques, with an appropriate sensitivity for the quantification of cocaine at the low concentrations present in some forensic samples. The cEIA was directly compared to LC-MS for the analysis of ten UK banknote samples. The results obtained from both techniques were statistically similar, suggesting that the immunoassay was unaffected by cross-reactivity with potentially interfering compounds. The cEIA was used also for the detection of cocaine in extracts from latent fingermarks. The results obtained were compared to the cocaine concentrations detected in oral fluid sampled from the same individual. Using the CEIA, we have shown, for the first time, that endogeneously excreted cocaine can be detected and quantified from a single latent fingermark. Additionally, it has been shown that the presence of cocaine, at similar concentrations, in more than one latent fingermark from the same individual can be linked with those concentrations found in oral fluid. These results show that detection of drugs in latent fingermarks could directly indicate whether an individual has consumed the drug. The specificity and feasibility of measuring low concentrations of cocaine in complex forensic samples demonstrates the effectiveness and robustness of the assay. The immunoassay
\end{abstract}


presents a simple and cost-effective alternative to the current mass spectrometry based techniques for the quantitation of cocaine at forensically significant concentrations.

Keywords: Cocaine, competitive enzyme immunoassay, quantification, banknotes, fingermarks.

\section{Introduction}

The supply and consumption of illicit drugs such as cocaine is a growing societal problem on a global scale. In order to combat the effect of usage and trade, many countries implement laws that allow the seizure of circumstantial evidence associated with cocaine trafficking [1].

It has been documented that a significant proportion of paper currency in general circulation is contaminated with cocaine [2-8]. Adherence of cocaine to the banknote can be due to surface contamination and entrapment of drug crystals between fibres of the note [9], while indirect transfer of cocaine is possible through contact with other notes or bank sorting machines [10]. Cocaine trading, using cash, and administration via a rolled up banknote can lead to direct exposure of banknotes to the crystalline drug powder [11]. It is thought that a concentration of cocaine on a note that is beyond that of the general circulation can be associated with drug use or trade and thus provide incriminating evidence $[12,13]$. Quantitative methods which enable the differentiation between 'drug money' and general currency, that are suitable for daily analysis in a forensic laboratory are crucial in providing such evidence. It is therefore of interest that a greater range of suitable methods for this type of analysis be developed.

Interest has also grown in recent years in gaining information from latent fingermarks beyond suspect identification. Additional information in a forensic context, such as the use by or recent exposure of an individual to illicit drugs, could provide enhanced evidence between a fingermark and a suspect. The successful detection of cocaine and other drug compounds in latent fingermarks is known using a number of different analytical approaches, and is particularly well-described for prints artificially doped with the compound of interest [14-17]. However, recent advances by our research group using an immuno-labelling approach based on the use of fluorescently labelled, antibody-functionalised magnetic particles have shown the detection of drug metabolites endogenously produced in the latent fingermarks of drug users [18-20]. In addition, Rowell et al. were able to show isolated patches of cocaine contamination in a fingerprint obtained from an individual being treated at a drug addiction centre using surface assisted laser desorption/ionisation time-of-flight mass spectrometry (SALDI-TOF-MS). The non-homogenous pattern of cocaine contamination in the print was 
thought to be due to the subject's fingertip coming into contact with the drug prior to the time of sampling [16]. Analytical methods for the detection of these types of cocaine residues in latent fingermarks could be used in a forensic context, as their presence would provide further evidence of a suspect's involvement in drug use or trade.

At present, gas chromatography, liquid chromatography or thermal desorption coupled with mass spectrometry (MS) are most often employed for the detection of cocaine extracted from banknotes $[4,5,8,11,21]$. Alternative methods include ion-mobility spectrometry [7, 22], electrochemiluminescence [23], and Raman microspectroscopy [9]. The immunodetection of cocaine on banknotes is limited to the application of strip-based immunoassays originally developed for qualitative detection of the drug in urine [24, 25]. The detection of cocaine extracted from doped fingerprints is similarly focused on MS-based approaches, such as desorption electrospray ionization (DESI)-MS [15] and SALDI-TOF-MS [16] or Raman spectroscopy [14, 17].

Immunoassays such as competitive enzyme immunoassays (cEIAs), where free analyte from a sample competes with a labelled analyte for available antibody binding sites on a micro-titer plate, are also commonly used for the detection of illicit compounds in biological samples such as blood and urine [26]. Assays of this nature offer excellent sensitivity and specificity in a simple to use and cost effective manner making them highly suited for forensic analysis, without requiring specialised chemicals or advanced instrumentation.

The aim of the present study was to develop a sensitive cEIA specifically designed for the quantitation of cocaine in forensic samples such as latent fingermarks and paper currency. Ten Bank of Scotland (UK) banknotes obtained from general circulation were tested for cocaine using the developed cEIA method as a preliminary indication of efficacy. The assay was validated by comparison with analysis of the same extracted samples by LC-MS. Further, the CEIA was used for the detection of cocaine in ten latent fingermarks. The fingermarks were obtained from five test subjects being treated for the habitual use of drugs, including cocaine, at a Methadone Treatment Clinic. The concentrations determined in the extracted fingermark samples were compared with the cocaine concentrations quantified by GC-MS in oral fluid samples collected from the same individuals. 


\section{Experimental}

\section{Materials}

All reagents were of analytical grade, purchased from Sigma-Aldrich (UK) and used as received unless otherwise stated. Polyclonal anti-cocaine antibody was obtained in $1 \mathrm{~mL}$ units of rabbit serum from Europa Bioproducts (UK) and purified as reported below. 'Slide-ALyzer' mini dialysis units (10,000 MWCO), 'NAb Protein A plus' spin columns, Coomassie brilliant blue R250, bromophenol blue sodium salt, ammonium persulfate, 96-well Nunc C8 Maxisorp microtiter plates and 3,3',5,5' tetramethylbenzidine (TMB) substrate solution, $50 \mathrm{~mL}$ centrifuge tubes and $1.5 \mathrm{~mL}$ Eppendorf microcentrifuge tubes were all purchased from Thermo Scientific (UK). Novex Sharp unstained protein standard was purchased from Invitrogen (UK), and N,N,N'-tetramethylethylenediamine (TEMED) was purchased from Bio Rad (UK). Cocaine standard (Cerilliant; $1 \mathrm{mg} \mathrm{mL}^{-1}$ in acetonitrile) and cocaine-horseradish peroxidase conjugates (cocaine-HRP) were purchased from LGC Standards (UK) and Randox (UK), respectively. Ten Scottish banknotes of $£ 10$ and $£ 20$ denominations from general circulation were kindly provided by the Scottish Crime and Drug Enforcement Agency (SCDEA). Ten fingermark samples on glass microscope slides were obtained from five volunteers attending a Methadone Treatment Clinic.

UV-VIS absorption spectra were recorded on a Hitachi U-3000 spectrophotometer. All centrifugation steps were performed using a Beckman Coulter Allegra X-22R centrifuge. Microtiter plate absorbance intensity readings were recorded using a Perkin Elmer Wallac Envision 2103 multilabel microplate reader. The confirmation analysis of the banknote extracts was performed using a Thermo LTQ Orbitrap Discovery high resolution accurate mass LC-MS running in full scan positive ion electrospray mode. The orbitrap was operating at a mass resolution of $30,000 \mathrm{FWHM}$ at $\mathrm{m} / \mathrm{z} 400$.

\section{Anti-cocaine antibody purification}

Polyclonal anti-cocaine antibody was isolated from the supplied rabbit serum samples by immunoprecipitation followed by Protein A chromatography. Ammonium sulfate precipitation was performed using standard methodology [27], prior to dialysis in mini-dialysis units for $2 \mathrm{~h}$ against $2 \mathrm{~L}$ of $10 \mathrm{mM}$ phosphate buffer ( $\mathrm{pH} 7.4$; PB). Immunochromatography was performed using Protein A spin columns with a sample capacity of $\leq 500 \mu \mathrm{L}$ in accordance with the manufacturer's instructions. Three columns were used to accommodate the $900 \mu \mathrm{L}$ of solution to be purified. Briefly, the columns were equilibrated before use by washing twice with $400 \mu \mathrm{L}$ of $10 \mathrm{mM}$ phosphate-buffered saline (150 mM NaCl, pH 7.2; PBS). Wash solutions were removed from the columns by centrifugation for $1 \mathrm{~min}$ at $4,000 \mathrm{xg}$. The 
solution containing the antibody was added at $300 \mu \mathrm{L}$ per column, and the columns incubated at room temperature on a rotary mixer for $10 \mathrm{~min}$. The columns were then washed three times with $400 \mu \mathrm{L}$ of $10 \mathrm{mM}$ PBS ( $\mathrm{pH}$ 7.2). The antibody was eluted from the columns using three $400 \mu \mathrm{L}$ additions of $0.1 \mathrm{M}$ glycine $(\mathrm{pH} 2.0)$. Each of the resulting three fractions was neutralised with $40 \mu \mathrm{L}$ of $1.0 \mathrm{M}$ tris-HCL buffer ( $\mathrm{pH}$ 8.5). The presence of antibody in the first and second fractions was determined by UV-VIS spectrophotometry at $A_{280}$ and confirmed by SDS-PAGE [28]. The two fractions were combined and dialysed in mini-dialysis units for $2 \mathrm{~h}$ against $2 \mathrm{~L}$ of $10 \mathrm{mM} \mathrm{PB}(\mathrm{pH} 7.4)$, and the solutions stored at $-20^{\circ} \mathrm{C}$.

\section{Banknote extraction}

Extracts from the banknotes were obtained using a modified version of the method described by Esteve-Turrillas et al. [3]. Each banknote was rolled and placed in a $20 \mathrm{~mL}$ glass test tube with $15 \mathrm{~mL}$ of methanol. The tube was sealed and vortex-mixed for $5 \mathrm{~min}$. The banknote was then removed from the tube and rinsed with a further $5 \mathrm{~mL}$ of methanol. The two extract volumes ( 15 and $5 \mathrm{~mL}$ ) were combined to make up the $20 \mathrm{~mL}$ final volume. The solvent was evaporated under reduced pressure at $40^{\circ} \mathrm{C}$ and the extract reconstituted in $0.5 \mathrm{~mL}$ of methanol. The reconstituted extract was centrifuged at $14,000 \mathrm{rpm}$ and $4{ }^{\circ} \mathrm{C}$ for $7 \mathrm{~min}$ to remove any solids while the supernatant was retained. Each extract was then divided into two halves; one half for CEIA and the other half for confirmation analysis by LCMS. Extract portions set aside for LC-MS confirmation were kept in methanol and stored at $4{ }^{\circ} \mathrm{C}$. For the immunoassay, $50 \mu \mathrm{L}$ of the extract was taken and the solvent was removed by drying under a gentle stream of argon. The extracts were then reconstituted in $50 \mu \mathrm{L}$ of $10 \mathrm{mM}$ PB ( $\mathrm{pH} 7.4$ ) containing $2 \% \mathrm{v} / \mathrm{v}$ methanol and stored at $4{ }^{\circ} \mathrm{C}$ overnight until analysed.

\section{Fingermark extraction}

Fingermarks were obtained from volunteers known to be taking illicit drugs. Each fingermark was deposited onto a clean glass microscope slide. The fingermarks were provided by the volunteers with no pre-treatment, i.e., the fingermarks contained a mixture of sebum and eccrine sweat. Oral fluid samples were collected from the volunteers at the same time and analysed by GC-MS. Fingermarks from drug-free volunteers were taken in the same manner as for the test subjects and used as negative controls. Full ethical permission was obtained prior to collection of samples. Cocaine was extracted from each fingermark separately using a similar method as described previously for the banknote samples. Specifically, the microscope slide with the fingermark was inserted into a $50 \mathrm{~mL}$ centrifuge tube, and $20 \mathrm{~mL}$ of methanol was added. The tube was sealed and vortex-mixed for $5 \mathrm{~min}$. The solvent was evaporated under reduced pressure at $40{ }^{\circ} \mathrm{C}$ and the extract reconstituted in $0.5 \mathrm{~mL}$ of 
$10 \mathrm{mM}$ PB (pH 7.4) containing $2 \% \mathrm{v} / \mathrm{v}$ methanol. The samples were stored at $4{ }^{\circ} \mathrm{C}$ overnight until analysed.

\section{Preparation of standard and sample solutions}

A cocaine standard stock solution ( $1 \mathrm{mg} \mathrm{mL}^{-1}$ ) was prepared in advance of analysis as follows. A $50 \mu \mathrm{L}$ aliquot of cocaine standard was taken and the acetonitrile removed by evaporation under a gentle stream of argon. The standard was then reconstituted in $50 \mu \mathrm{L}$ of $10 \mathrm{mM}$ PB ( $\mathrm{pH}$ 7.4) containing $2 \% \mathrm{v} / \mathrm{v}$ acetonitrile. A standard curve was prepared on the day of analysis by diluting the stock solution to known concentrations in $10 \mathrm{mM} \mathrm{PB} \mathrm{(pH} \mathrm{7.4).}$ The concentrations of cocaine for each curve were as follows; $0.78,1.56,3.125,6.25,12.5$, 25,50 and $100 \mathrm{ng} \mathrm{mL}^{-1}$ for the analysis of banknote extracts, and $0.195,0.39,0.78,1.56$, $3.125,6.25,12.5$, and $25 \mathrm{ng} \mathrm{mL}^{-1}$ for the analysis of fingermark extracts. Banknote extracts were diluted to $1: 500$ by adding $1 \mu \mathrm{L}$ of sample to $499 \mu \mathrm{L}$ of $10 \mathrm{mM} \mathrm{PB}(\mathrm{pH} 7.4)$, or to $1: 1000$ by adding $1 \mu \mathrm{L}$ of sample to $999 \mu \mathrm{L}$ of PB immediately prior to analysis. Fingermark extracts were not diluted prior to analysis.

\section{Anti-cocaine competitive enzyme immunoassay (cEIA)}

A schematic overview of the developed cEIA method is shown in Fig. 1. The assay involved the adsorption of the purified anti-cocaine antibody $\left(1 \mu \mathrm{g} \mathrm{mL}^{-1}\right)$ to a microtiter plate at $100 \mu \mathrm{L}$ per well. During initial development, a comparison between $10 \mathrm{mM} \mathrm{PB}(\mathrm{pH} 7.4)$ and $100 \mathrm{mM}$ carbonate buffer ( $\mathrm{pH}$ 9.5) showed greater adsorption of antibody occurred when the carbonate buffer was used as a diluent. Therefore, adsorption using antibody diluted in carbonate buffer was used thereafter. The plate containing the antibody $\left(1 \mu \mathrm{g} \mathrm{mL}^{-1}\right.$ in carbonate buffer) was incubated overnight $(18 \mathrm{~h})$ at $4{ }^{\circ} \mathrm{C}$ and then washed three times with 10 mM PBS containing $0.05 \%$ v/v Tween-20 (100 mM NaCl, pH 7.4; PBS/T). Blocking buffer, PBS containing $1 \% \mathrm{w} / \mathrm{v}$ bovine serum albumin (PBS/BSA), was added at $200 \mu \mathrm{L}$ per well and incubated at room temperature for $2 \mathrm{~h}$. The plate was then washed a further three times with PBS/T before the addition of pre-diluted standards and extracted samples (in PB) at $50 \mu \mathrm{L}$ per well. Following the addition of sample and standard solutions the plate was incubated for $2 \mathrm{~h}$ at room temperature. After incubation, $50 \mu \mathrm{L}$ of the cocaine-HRP conjugate, at varying dilutions in blocking buffer containing $0.05 \% \mathrm{v} / \mathrm{v}$ Tween-20, was added to each well. The solutions were mixed by gently tapping the side of the plate several times and then incubated for $2 \mathrm{~h}$ at room temperature. The plate was then washed four times with PBS/T. TMB substrate solution was added at $100 \mu \mathrm{L}$ per well followed by a $20 \mathrm{~min}$ incubation at room temperature in the absence of light. Sulfuric acid $(0.5 \mathrm{M})$ was added at $100 \mu \mathrm{L} /$ well to stop the enzymatic reaction, after which the absorbance of each well was 
measured at $A_{450}$ using the multi-label micro-plate reader. Wells without antibody were used as a blank. The intensity of the absorbance measured was inversely proportional to the quantity of cocaine in the extracted sample.

Absorbance intensities were corrected for cocaine-HRP binding without the presence of cocaine by dividing the signal of the sample or standard solution $(B)$ by that of the zero concentration calibrator $\left(\mathrm{B}_{0}\right)$. The standard curve was generated by plotting the cocaine concentration (on a logarithmic scale) against the logit of the corrected absorbance intensity $\left(\operatorname{Logit}\left(B / B_{0}\right)\right)$. For this the overall formula Logit $\left(B / B_{0}\right)=\left[\ln \left(\left(B / B_{0}\right) /\left(1-\left(B / B_{0}\right)\right)\right)\right]$ was applied [29]. 


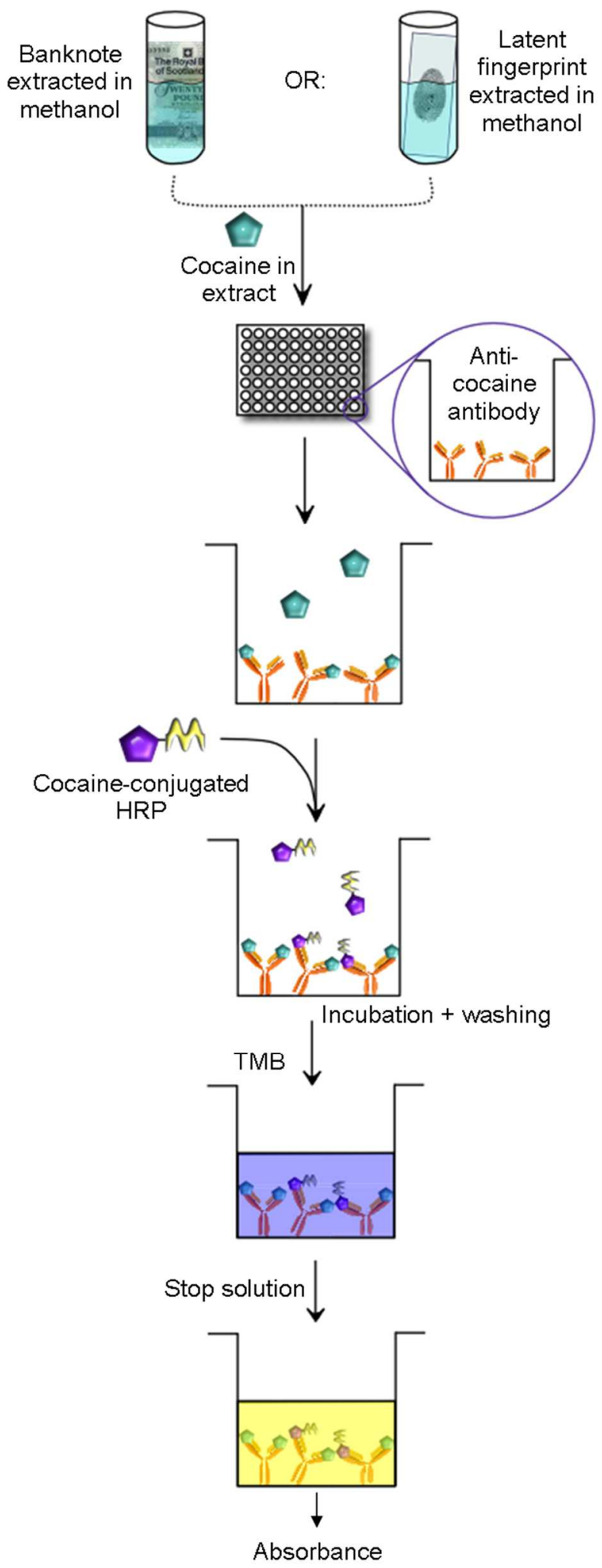

Fig. 1 A schematic representation of the CEIA for the detection of cocaine in extracted samples from banknotes or latent fingermarks. 


\section{Results and discussion}

\section{cEIA calibration, detection limit and precision}

Typical calibration curves for cocaine using the cEIA are shown in Fig. 2A and 2B. These curves were generated using cocaine standard in a buffer solution at concentrations ranging from 0.78 to $100.00 \mathrm{ng} \mathrm{mL}^{-1}$, and 0.195 to $25.00 \mathrm{ng} \mathrm{mL}^{-1}$ for the analysis of banknote and fingermark extracts, respectively. The data showed a good correlation coefficient $\left(r^{2}=0.999\right)$ on the logit-log plot using a linear regression for both calibration curves (Fig. 2C and 2D). The detection limit of the cEIA was calculated from the average absorbance values from six replicate analyses of the blank minus three times the standard deviation of the average. The resulting absorbance value was then extrapolated from the log-logit standard curve. The minimum detectable concentration of cocaine was determined as $0.162 \mathrm{ng} \mathrm{mL}^{-1}$. This detection limit compares favourably to the $0.95 \mathrm{ng} \mathrm{mL}^{-1}$ limit reported for the detection of cocaine and metabolites in oral fluid using a commercial micro-plate EIA [30], and to the $1.0 \mathrm{ng} \mathrm{mL}^{-1}$ achieved using GC-MS with solid phase extraction for the detection of cocaine on banknote samples [6]. 

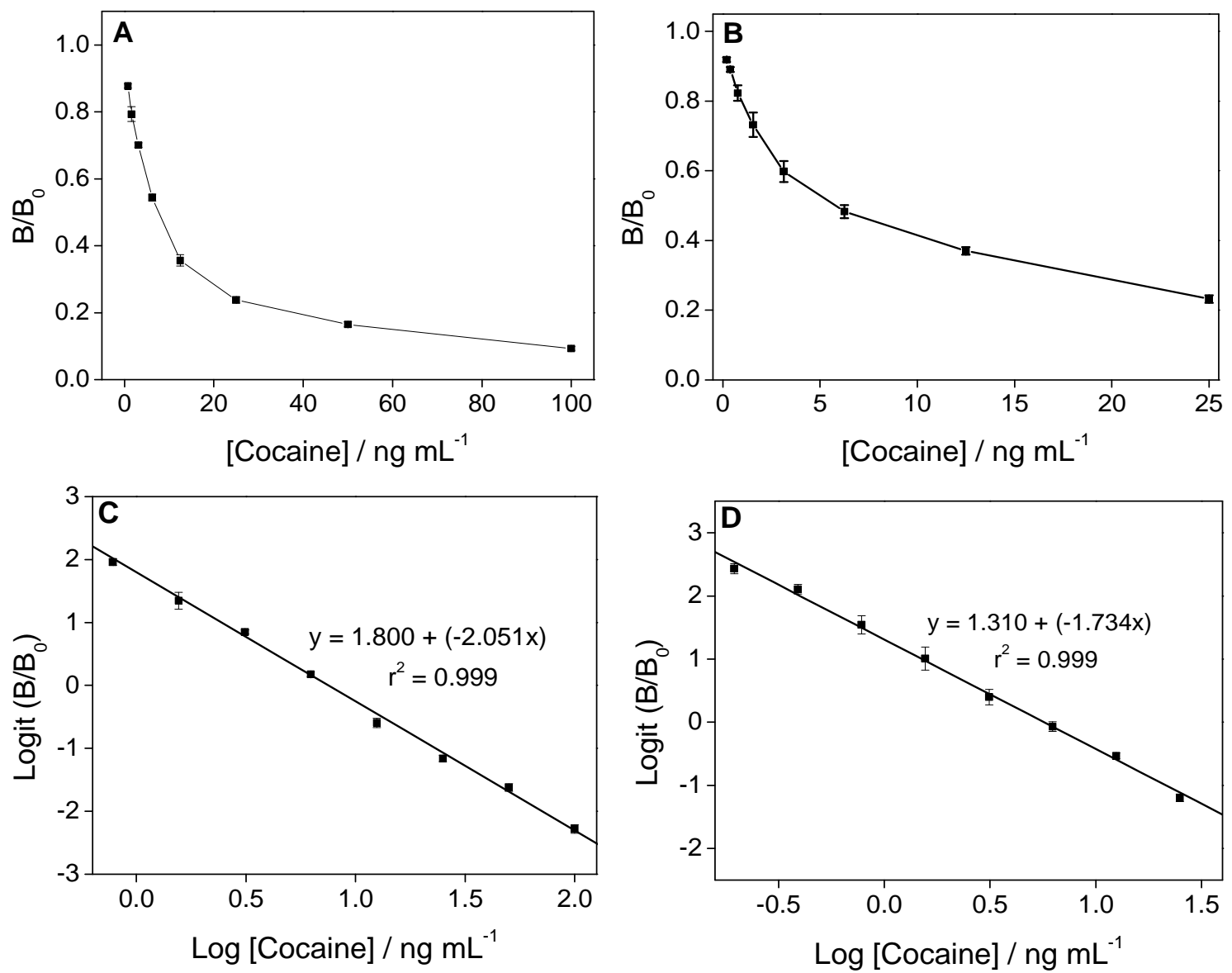

Fig. 2 Typical standard curves showing the corrected absorbance response $\left(B / B_{0}\right)$ as a function of cocaine concentration in the range of: (A) 0.78 to $100.00 \mathrm{ng} \mathrm{mL}^{-1}$ for banknote analysis; and (B) 0.195 to $25.00 \mathrm{ng} \mathrm{mL}^{-1}$ for fingermark analysis. Each point represents the mean \pm SD of at least three measurements. The corresponding logit-log linear calibration curves are shown for banknote and fingermark analysis in (C) and (D), respectively.

The precision of the assay was estimated based on repeat measurements of standard cocaine samples prepared in assay buffer (10 mM PB, pH 7.4). Standards at three different cocaine concentrations $\left(6.25,25\right.$ and $\left.100 \mathrm{ng} \mathrm{mL}^{-1}\right)$ were used. The intra-assay precision was determined using four replicates of each standard in a single analysis $(n=4)$. Inter-assay precision was similarly assessed by analysing the same sample, as four repetitions, across three separate analyses undertaken on different days over a period of 30 days $(n=12)$. The results presented in Table 1 highlights the precision of the developed cEIA for the quantitative detection of cocaine, with confidence variables of $1.58-6.54 \%$ and $4.38-6.69 \%$ for intra- and inter-assay precision, respectively. 
Table 1 Precision of the cEIA for the quantification of cocaine.

\begin{tabular}{llcllc}
\hline & \multicolumn{2}{l}{ Intra-assay $(\mathrm{n}=4)$} & & \multicolumn{2}{l}{ Inter-assay $(\mathrm{n}=12)$} \\
\cline { 2 - 3 } \cline { 5 - 6 } Cocaine $\left(\mathrm{ng} \mathrm{mL}^{-1}\right)$ & $\begin{array}{l}\text { Mean } \pm \mathrm{SD} \\
\left(\mathrm{ng} \mathrm{mL}^{-1}\right)\end{array}$ & $\mathrm{CV}^{\mathrm{a}}(\%)$ & & $\begin{array}{l}\text { Mean } \pm \mathrm{SD} \\
\left(\mathrm{ng} \mathrm{mL}^{-1}\right)\end{array}$ & $\mathrm{CV}^{\mathrm{a}}(\%)$ \\
\hline 6.25 & $6.100 \pm 0.097$ & 1.58 & & $6.363 \pm 0.279$ & 4.38 \\
25.0 & $25.119 \pm 0.894$ & 3.56 & & $25.849 \pm 1.207$ & 4.67 \\
100.0 & $98.723 \pm 6.456$ & 6.54 & & $98.416 \pm 6.587$ & 6.69 \\
\hline
\end{tabular}

${ }^{\mathrm{a}} \mathrm{CV}$ is the coefficient of variation.

\section{Evaluation of cEIA performance with real banknote samples}

UK banknotes comprise cotton and linen paper [12]. The way in which paper currency is handled and transferred means that a number of contaminants for example; dust, soil, food particles and cosmetics, can all be found on the fibres of a banknote. At a trace level, predominantly caffeine, nicotine, paracetamol and other pharmaceutical compounds have been reported on banknotes [10]. Drugs such as cocaine and its metabolite benzoylecgonine, tetrahydrocannabinol (THC), heroin, amphetamines, and ketamine have also been detected [1].

Typically, cocaine is extracted from banknotes using an organic solvent. For chromatographic analyses, this is followed by a form of sample clean-up such as centrifugation or filtration [13]. Such steps can be avoided with immunochemical analysis simply by diluting the sample in buffer prior to analysis [31]. Optimising immunoassays for pre-diluted samples lessens possible matrix effects by reducing the overall concentration of potential interferents in the sample, while maintaining an analyte concentration that is within the working range of the assay.

As highlighted, extracts from banknotes could contain a number of potential immunoassay interferents. Consequently, the influence of the chemical 'matrix effect' on the cEIA was determined. The general practise to test matrix effects involves spiking blank sample matrices with an appropriate standard at a known concentration and thus determining the accuracy of the assay results. As the majority of banknotes in general circulation are reported to be contaminated with cocaine such notes could not be used for spiking. Previously uncirculated banknotes were also avoided as they lack the appropriate 'handling and transfer' contaminants to properly represent the sample matrix. Instead, cocaine was 
extracted from ten UK banknote samples using a modified version of the method described by Esteve-Turrillas et al. [3]. Esteve-Turrillas et al. reported recoveries of $101 \pm 2$ and $98 \pm$ $3 \%$ in spiked banknote samples extracted using their method followed by GC-MS-MS analysis.

The extracts from the banknote samples were each subjected to two dilution factors (1:500 and 1:1000) and analysed by the CEIA. The cocaine concentrations were corrected for the dilution factor and compared to determine what effect doubling the optimised concentration of raw extract would have on the accuracy of the assay. Results showing the change in absorbance relative to cocaine concentration in standard solutions and the absorbance response to the two extract concentrations are shown in Fig. $3 \mathrm{~A}$ and $3 \mathrm{~B}$, respectively. The values shown in Fig. $3 A$ were used to generate the standard curve from which the cocaine concentrations obtained from the ten banknote samples were calculated (Fig. 3B and Table 2). The average difference in cocaine concentration between the two extract dilutions across all of the ten banknote samples was $\pm 0.810 \mu \mathrm{g} \mathrm{mL}^{-1}$. The differences ranged from \pm 0.037 to $1.915 \mu \mathrm{g} \mathrm{mL}^{-1}$. The variation in concentration between the two sample dilutions showed that the matrix does affect the response of the assay, although the variation between the two dilutions did not exceed the standard deviation obtained from the repeat measurements (results not shown). An overall increase in the absorbance signal was obtained using the more dilute (1:1000) rather than the more concentrated (1:500) samples, suggesting that the 1:1000 dilution provides a more appropriate matrix for the cEIA. Based on this, the quantitative results obtained using the 1:1000 sample dilution were applied to the parallel analysis using LC-MS. 


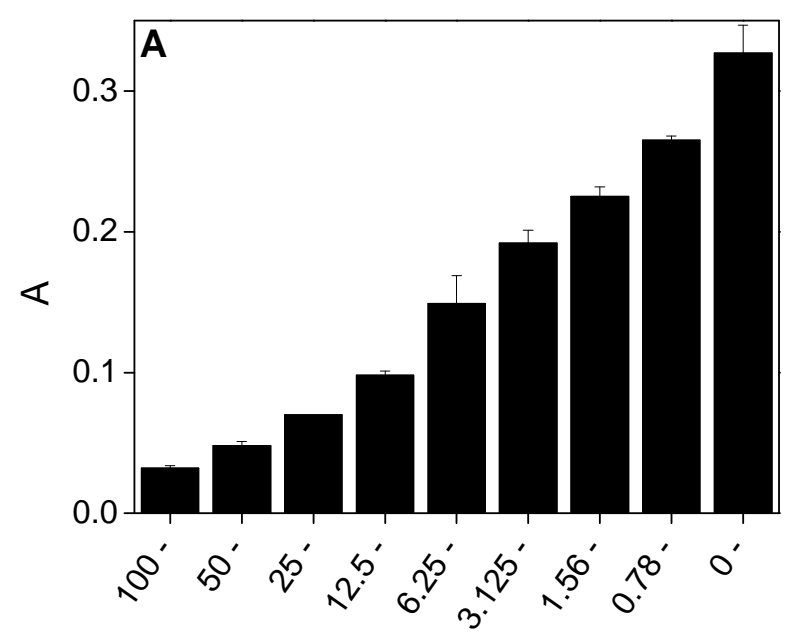

[Cocaine] $/ \mathrm{ng} \mathrm{mL}^{-1}$

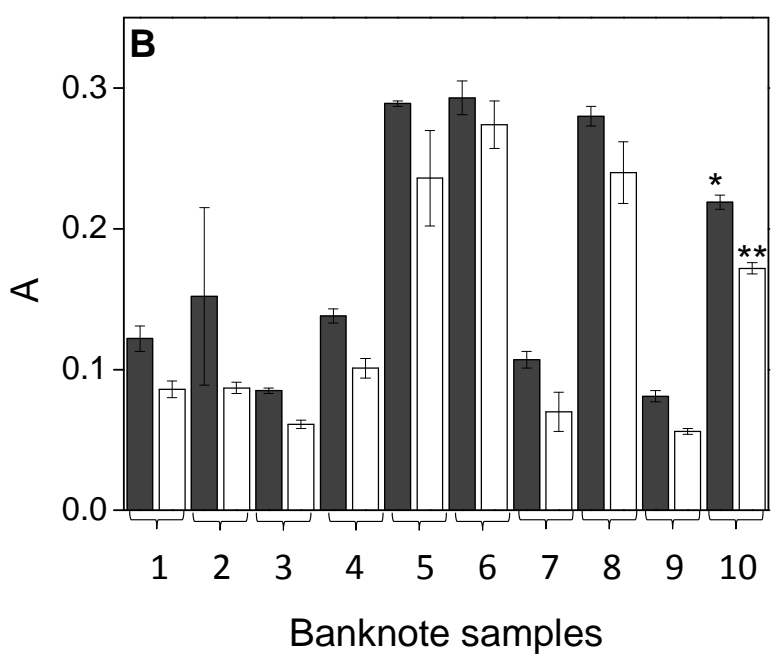

Banknote samples

Fig. 3 The absorbance intensity obtained (A) using the cEIA from a series of cocaine standard solutions, and (B) from ten extracted banknote samples. The ten banknote samples were diluted to a factor of $1: 1000$ and 1:500. The results from the two dilutions of each banknote sample are shown in the order of lowest extract concentration to highest. As an example, the $1: 1000$ and 1:500 dilutions of Sample 10 are labelled $\left({ }^{*}\right)$ and $\left({ }^{* *}\right)$, respectively.

Each absorbance value represents the mean \pm SD of at least four measurements.

The results obtained from the cEIA for the banknote extracts were compared with those obtained by a conventional LC-MS method. Extracts from the ten UK banknote samples were obtained as described and analysed using the two techniques using the same cocaine standard as a reference. The LC-MS analysis was performed using the method described by Hudson and Timber [32]. Table 2 shows the concentration values obtained using the cEIA and LC-MS. Using a paired t-test it was shown that the concentrations determined using the two techniques did not differ significantly from one another at $P=0.1$. Benzoylecgonine $(B E)$, a major metabolite of cocaine, and other compounds of structural similarity were detected by the LC-MS in the extracted samples (results not shown). Despite the presence of these potential interferents, the cEIA yielded cocaine concentrations similar to those detected by LC-MS. The agreement between the results of the immunoassay and the LCMS analysis suggests that the assay was not affected by cross-reactivity when applied to the analysis of cocaine in real banknote samples. The results show that the developed cEIA therefore enables the quantitation of cocaine in complex banknote extracts with a degree of sensitivity that is comparable to the current chromatographic-mass spectrometry technique. 

samples undertaken using the $c E I A^{a}$ and LC-MS.

\begin{tabular}{llc}
\hline Sample & $\begin{array}{l}\mathrm{CEIA}, \\
\mu \mathrm{mL}^{-1}\end{array}$ & $\begin{array}{l}\mathrm{LC}-\mathrm{MS}, \\
\mu \mathrm{mL}^{-1}\end{array}$ \\
\hline 1 & $9.322 \pm 1.655$ & 4.181 \\
2 & $7.317 \pm 4.233$ & 10.026 \\
3 & $19.131 \pm 1.031$ & 13.044 \\
4 & $6.921 \pm 0.553$ & 4.699 \\
5 & $0.385 \pm 0.025$ & 0.448 \\
6 & $0.297 \pm 0.143$ & 0.245 \\
7 & $11.945 \pm 1.320$ & 8.583 \\
8 & $0.467 \pm 0.107$ & 0.529 \\
9 & $19.950 \pm 1.567$ & 13.780 \\
10 & $1.893 \pm 0.177$ & 2.205 \\
\hline
\end{tabular}

aEach cEIA value represents the mean \pm SD of at least three measurements.

\section{Application of the cEIA for the analysis of latent fingermarks}

Two possibilities exist for the way in which latent fingermarks can become contaminated with cocaine. The first of these is through contact transfer, known to occur when the fingertip of an individual comes into contact with the crystalline drug powder. The crystals of cocaine stick to the sweaty skin of the fingertip for a short time, and via the sweat are transferred to surfaces that the skin comes into contact with. The second route for contamination of fingermarks is as a result of the individual taking the drug itself. When a drug enters the body it is transported via the circulation to the liver, where it is metabolised, transported to the kidneys and then excreted in the urine. During this process, drugs and drug metabolites can be secreted onto the skin surface from the circulation through the action of sweating [33]. Hazarika et al. have shown that drug metabolites such as benzoylecgonine, a major metabolite of cocaine, were present at a detectable level in the fingermarks of cocaine users $[18,19]$. In addition, the use of SALDI-TOF-MS enabled the detection of EDDP, a major metabolite of methadone, together with its parent drug in the same fingermark from an individual taking methadone [16]. As methadone is taken as an oral dose, it was concluded that the presence of the parent drug in the fingermark arises from its secretion from the blood in the same manner as for metabolites. The presence and quantitation of cocaine at a detectable level in the sweat of a fingermark from a known cocaine user has not previously been reported.

Several studies have shown a strong correlation between cocaine concentration in oral fluid and in plasma, proving oral fluid to be an appropriate sample matrix for the detection of drug 
use $[34,35]$. Due to its rapid elimination, with a half-life of only $3 \mathrm{~h}$, the detection of cocaine in oral fluid is a suitable indicator of recent drug use close to the time of sampling [36]. In this study, oral fluid and latent fingermark samples were taken at the same time from five individuals attending a Methadone Treatment Clinic. Two fingermark samples were taken per subject and extracted and analysed using the developed cEIA method. The results were compared with the results of GC-MS analysis of the corresponding oral fluid samples.

The concentrations of cocaine determined in the oral fluid samples by GC-MS, and the extracted fingermarks by cEIA are shown in Fig $4(A)$ and $(B)$, respectively. Cocaine was detected in oral fluid samples from three of the five test subjects, with the sample from subject P2 containing the highest concentration (19 $\left.\mathrm{ng} \mathrm{mL}^{-1}\right)$. This corresponds to the highest concentration of cocaine determined using the cEIA in both fingermarks from subject $\mathrm{P} 2$ at 0.728 and $0.879 \mathrm{ng} \mathrm{mL}^{-1}$ for (a) and (b) samples, respectively. The oral fluid samples from subjects $\mathrm{P} 1$ and $\mathrm{P} 3$ also tested positive for cocaine. These positive cocaine tests correspond to positive fingermark samples, although the relative concentrations are different. The fingermarks from subject P4 gave cocaine results where one print was significantly higher than the other print. Since the oral fluid result from volunteer P4 was negative, contamination by contact transfer could possibly account for this result. The fingermark samples P4(a), both prints from P5 and the negative control samples (P6 and P7) did not contain cocaine at detectable concentrations based on the sensitivity of the assay.

The overall trend of the data shows that the cocaine present in the latent fingermark samples as determined by the CEIA reflects that of the oral fluid concentrations, particularly as the concentrations detected were similar in both of the fingermark samples from the same subject. The developed cEIA method proved to be readily applicable for the analysis of these types of samples, even at cocaine concentrations within the fingermark extract of less than $1 \mathrm{ng} \mathrm{mL}^{-1}$. As the presence of the drug in oral fluid is a recognised indicator of recent cocaine use, the results obtained suggest, for the first time, that the detection of cocaine in the sweat of a latent fingermark could be similarly linked to consumption. 

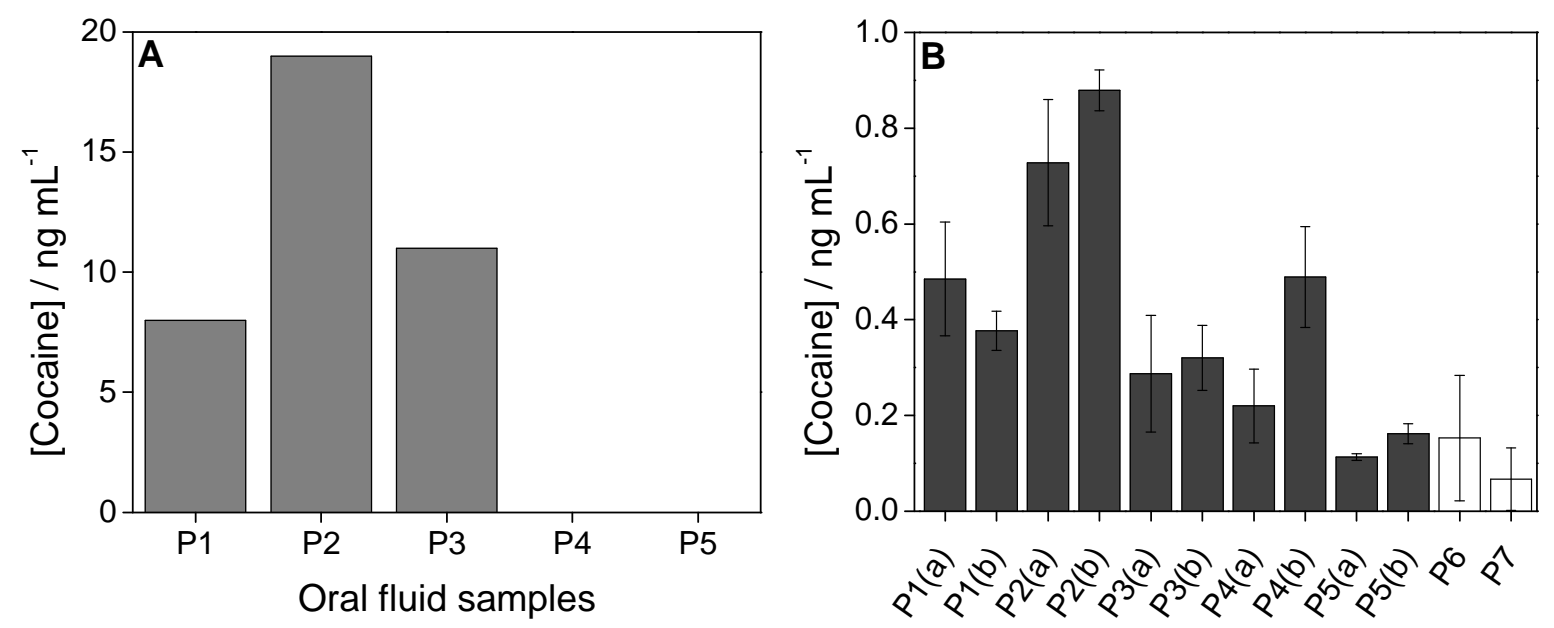

Fingerprint samples

Fig. 4 (A) Results of a quantitative analysis of cocaine by GC-MS on oral fluid samples taken from five individuals (numbered P1 - P5) from a methadone maintenance clinic, and (B) results of the CEIA analysis of cocaine extracted from fingermarks of individuals P1-P5, taking two fingermarks (samples $a$ and $b$ ) from each individual. The first ten fingermarks, P1-

P5, correspond to the individuals from which oral fluid was also taken. P6 and P7 were single prints obtained from two separate drug-free individuals as negative controls. Each concentration value represents the mean \pm SD of at least three measurements.

\section{Conclusions}

In this study, a non-destructive and sensitive competitive enzyme immunoassay (cEIA) enabling the quantitation of cocaine in complex forensic samples such as extracts from banknotes and latent fingermarks has been developed. The level of sensitivity achieved by the assay, with a LOD of $0.162 \mathrm{ng} \mathrm{mL}^{-1}$, enables the quantitation of cocaine in samples of forensic significance. The quantification of cocaine from ten UK banknote samples was performed using the cEIA and compared by parallel LC-MS analysis. The results from both methods were found to be in agreement, indicating that the cEIA has the required specificity for the analysis of complex, heavily contaminated banknote extracts, and is not affected by the presence of BE and other potential interferents in the samples. Trace levels of cocaine were detected from all ten banknotes sampled using the assay. The concentrations detected were in agreement with the background levels of cocaine detected on Irish Euro banknotes in general circulation [5].

The cEIA was similarly applied to the quantification of cocaine extracted from latent fingermarks donated by five individuals attending a Methadone Treatment Clinic. The results 
were compared to the cocaine concentrations determined by GC-MS in oral fluid samples obtained from the test subjects at the time the fingermarks were taken. The overall trend of the data showed, for the first time, that the cocaine detected at similar concentrations in more than one latent fingermark from the same individual followed the same pattern as those in oral fluid. The results suggest that the detection of drugs from fingermarks could be associated with consumption. The developed cEIA offers a straight forward, cost-effective alternative to chromatography-MS techniques for the quantitation of cocaine in forensic samples, without the need for specialised instrumentation. The simplicity of the developed cEIA provides a method that would be readily applicable for everyday analysis in a forensic laboratory.

\section{Acknowledgements}

The authors acknowledge the School of Chemistry, University of East Anglia for the studentship for S.v.d.H. The Home Office Centre for Applied Science and Technology (CAST) and Intelligent Fingerprinting Ltd. are gratefully acknowledged for their financial support. The authors are grateful to the Scottish Crime and Drug Enforcement Agency (SCDEA) for providing the UK banknote samples and Intelligent Fingerprinting Ltd. for providing the oral fluid analysis data used in this study. 


\section{References}

[1] R. Sleeman, F. Burton, J. Carter, D. Roberts, P. Hulmston, Drugs on money, Anal. Chem. 72 (2000) 397A-403A.

[2] O. P. Luzardo, M. Almeida, M. Zumbado, L.D. Boada, Occurrence of contamination by controlled substances in Euro banknotes from the Spanish Archipelago of the Canary Islands, J. Forensic Sci. 56 (2011) 1588-1593.

[3] F. A. Esteve-Turrillas, S. Armenta, J. Moros, S. Garrigues, A. Pastor, M. de la Guardia, Validated, non-destructive and environmentally friendly determination of cocaine in Euro bank notes. J.

Chromatogr. A 1065 (2005) 321-325.

[4] K. Wimmer, S. Schneider, Screening for illicit drugs on Euro banknotes by LC-MS/MS, Forensic Sci. Int. 206 (2011) 172-177.

[5] J. Bones, M. Macka, B. Paull, Evaluation of monolithic and sub $2 \mu \mathrm{m}$ particle packed columns for the rapid screening for illicit drugs - application to the determination of drug contamination on Irish Euro banknotes, Analyst 132 (2007) 208-217.

[6] A. J. Jenkins, Drug contamination of US paper currency, Forensic Sci. Int. 121 (2001) 189-193.

[7] F. Anglada, O. Delémont, O. Guéniat, P. Esseiva, Detection and significance of cocaine traces on Swiss and Euro banknotes, CHIMIA 66 (2012) 346.

[8] E. Di Donato, C. C. S. Martin, B. S. De Martinis, Determination of cocaine in Brazilian paper currency by capillary gas chromatography/mass spectrometry, Quim. Nova. 30 (2007) 1966-1967. [9] K. A. Frederick, R. Pertaub, N. W. Shi Kam, Identification of individual drug crystals on paper currency using Raman microspectroscopy, Spectrosc. Lett. 37 (2004) 301-310.

[10] J.F. Carter, R. Sleeman, J. Parry, The distribution of controlled drugs on banknotes via counting machines, Forensic Sci. Int. 132 (2003) 106-112.

[11] K. A. Ebejer, G. R. Lloyd, R. G. Brereton, J. F. Carter, R. Sleeman, Factors influencing the contamination of UK banknotes with drugs of abuse, Forensic Sci. Int. 171 (2007) 165-170.

[12] K. A. Ebejer, J. Winn, J. F. Carter, R. Sleeman, J. Parker, F. Körber, The difference between drug money and a "lifetime's savings", Forensic Sci. Int. 167 (2007) 94-101.

[13] S. J. Dixon, R. G. Brereton, J. F. Carter, R. Sleeman, Determination of cocaine contamination on banknotes using tandem mass spectrometry and pattern recognition, Anal. Chim. Acta. 559 (2006) 54-63.

[14] J. S. Day, H. G. Edwards, S. A. Dobrowski, A. M. Voice, The detection of drugs of abuse in fingerprints using Raman spectroscopy I: latent fingerprints. Spectrochim. Acta A 60 (2004) 563-568. [15] D. R. Ifa, N. E. Manicke, A. L. Dill, R. G. Cooks, Latent fingerprint chemical imaging by mass spectrometry, Science 321 (2008) 805.

[16] F. Rowell, K. Hudson, J. Seviour, Detection of drugs and their metabolites in dusted latent fingermarks by mass spectrometry, Analyst 134 (2009) 701-707.

[17] M. J. West, M. J. Went, The spectroscopic detection of drugs of abuse in fingerprints after development with powders and recovery with adhesive lifters, Spectrochim. Acta A 71 (2009) 19841988.

[18] P. Hazarika, S. M. Jickells, K. Wolff, D. A. Russell, Imaging of latent fingerprints through the detection of drugs and metabolites, Angew. Chem. Int. Edit. 47 (2008) 10167-10170.

[19] P. Hazarika, S. M. Jickells, K. Wolff, D. A. Russell, Multiplexed detection of metabolites of narcotic drugs from a single latent fingermark, Anal. Chem. 82 (2010) 9150-9154.

[20] P. Hazarika, D. A. Russell, Advances in fingerprint analysis. Angew. Chem. Int. Edit. 51 (2012) 3524-3531.

[21] S. Armenta, M. de la Guardia, Analytical methods to determine cocaine contamination of banknotes from around the world, Trac-Trends Anal. Chem. 27 (2008) 344-351.

[22] F. E. Dussy, C. Berchtold, T. A. Briellmann, C. Lang, R. Steiger, M. Bovens, Validation of an ion mobility spectrometry (IMS) method for the detection of heroin and cocaine on incriminated material, Forensic Sci. Int. 177 (2008) 105-111. 
[23] Q. Cai, L. Chen, F. Luo, B. Qiu, Z. Lin, G. Chen, Determination of cocaine on banknotes through an aptamer-based electrochemiluminescence biosensor, Anal. Bioanal. Chem. 400 (2011) 289-294. [24] R. Wennig, M. R. Moeller, J. M. Haguenoer, A. Marocchi, F. Zoppi, B. L. Smith, R. de la Torre, C. A. Carstensen, A. Goerlach-Graw, J. Schaeffler, R. Leinberger, Development and evaluation of immunochromatographic rapid tests for screening of cannabinoids, cocaine, and opiates in urine. J Anal. Toxicol. 22 (1998) 148-155.

[25] P. Shrivastava, C. J. McLean, F. Aberl, J. Bonenberger, R.-P. Berg, R. Zimmermann, Immunosensor-based drug detectors for customs and other operations, Proc. SPIE 2937, 183 (1997) 183-190.

[26] S. Carney, C. E. Wolf, L. Tarnai-Moak, A. Poklis, Evaluation of two enzyme immunoassays for the detection of the cocaine metabolite benzoylecgonine in 1,398 urine specimens, J. Clin. Lab. Anal. 26 (2012) 130-135.

[27] M. Page, R. Thorpe, Purification of IgG by precipitation with sodium sulfate or ammonium sulfate, in: J. M. Walker (Ed.), The Protein Protocols Handbook, Springer, New York, 1996, pp. 721722.

[28] J. M. Walker, SDS polyacrylamide gel electrophoresis of proteins, in: J. M. Walker (Ed.), The Protein Protocols Handbook, third ed., Springer, New York, 2009, pp. 177-185.

[29] D. Wild, The Immunoassay Handbook, fourth ed., Elsevier Science Limited, Oxford, 2013.

[30] R. S. Niedbala, K. Kardos, T. Fries, A. Cannon, A. Davis, Immunoassay for detection of cocaine/metabolites in oral fluids, J. Anal. Toxicol. 25 (2001) 62-68.

[31] G. B. Wisdom, Enzyme-immunoassay, Clin. Chem. 22 (1976) 1243-1255.

[32] S. M. Hudson, S. Timbers, Proceedings of the 18th International Conference of Racing Analysts and Veterinarians, New Zealand, Dunmore Publishing Ltd, 2010, pp. 133-141.

[33] D. A. Kidwell, J. C. Holland, S. Athanaselis, Testing for drugs of abuse in saliva and sweat, J. Chromatogr. B 713 (1998) 111-135.

[34] V. Vindenes, H. Lund, W. Andresen, H. Gjerde, S. Ikdahl, A. Christophersen, E. L. Øiestad, Detection of drugs of abuse in simultaneously collected oral fluid, urine and blood from Norwegian drug drivers, Forensic Sci. Int. 219 (2012) 165-171.

[35] W. M. Bosker, M. A. Huestis, Oral fluid testing for drugs of abuse, Clin. Chem. 55 (2009) 19101931.

[36] K. B. Scheidweiler, E. A. K. Spargo, T. L. Kelly, E. J. Cone, A. J. Barnes, M. A. Huestis, Pharmacokinetics of cocaine and metabolites in human oral fluid and correlation with plasma concentrations following controlled administration, Ther. Drug Monit. 32 (2010) 628-637. 